CORRIGENDUM

doi:10.1038/nature13877

\title{
Corrigendum: Connectomic reconstruction of the inner plexiform layer in the mouse retina.
}

Moritz Helmstaedter, Kevin L. Briggman, Srinivas C. Turaga, Viren Jain, H. Sebastian Seung \& Winfried Denk

Nature 500, 168-174 (2013); doi:10.1038/nature12346

It has been brought to our attention that Supplementary Data 7 , reporting the correspondence of our cell type definitions to those reported in the literature, contained sorting errors in the first two columns of the table. The correct table is shown in the Supplementary Information to this Corrigendum.

We would also like to clarify that although some authors distinguish between the amacrine cell types A2 (receiving cone bipolar input) and AII (part of the rod bipolar pathway), we have used 'A2' in reference to the amacrine cell of the rod bipolar pathway, only. Furthermore, our classification of cone bipolar cell types $\mathrm{CBC} 1$ and $\mathrm{CBC} 2$ needs to be treated with caution. To distinguish $\mathrm{CBC} 1$ from $\mathrm{CBC} 2$ we primarily used the width of axonal stratification along the light axis, with $\mathrm{CBC} 1$ as the wider cells (on the basis of the morphological sketch in ref. 1 (ref. 28 in original Article)), and the mosaic fit as a secondary criterion. We used the prevalence reported in ref. 1, and found reasonable but not perfect mosaics for both $\mathrm{CBC} 1$ and $\mathrm{CBC} 2$ cells, and we interpreted this as confirmation of our choice of assignment. We have, however, since discovered that starting with the converse assumption, that the narrower cells correspond to $\mathrm{CBC} 1$ rather than $\mathrm{CBC} 2$, but otherwise proceeding in the same way we find mosaics of similar quality. The alternative sorting of cells is: CBC1 ( $n=26$, matrix IDs 393, 400, 403, 410, 412, 414, 415, 417, 418, 420, 421, 422, 424, 427, 430, 431, 432, 433, 436, 438, 440, 441, 444, 445, $447,449)$; CBC2 ( $n=34$, matrix IDs 390, 391, 392, 394, 395, 396, 397, 398, 399, 401, 402, 404, 405, 406, 407, 408, 409, 411, 413, 416, 419, 423, $425,426,428,429,434,435,437,439,442,443,446,448)$. In fact, on the basis of the degree of axonal overlaps that remains with either sorting we cannot rule out the possibility that there may be a third sparse bipolar cell type among the CBC1 and CBC2 cells.

Supplementary Information is available in the online version of this Corrigendum.

1. Wässle, H., Puller, C., Muller, F. \& Haverkamp, S. Cone contacts, mosaics, and territories of bipolar cells in the mouse retina. J. Neurosci. 29, 106-117 (2009). 